\title{
Gycyrrhizic acid alleviates atherosclerotic lesions in rats with diabetes mellitus
}

\author{
YAODONG ZHAO ${ }^{1}$, WEI LI ${ }^{1}$ and DAIMIN ZHANG ${ }^{2}$ \\ ${ }^{1}$ Department of General Internal Medicine, The Fifth Affiliated Hospital of Zhengzhou University, Zhenzhou, Henan 450052; \\ ${ }^{2}$ Department of Cardiology, Nanjing First Hospital, Nanjing Medical University, Nanjing, Jiangsu 210006, P.R. China
}

Received July 31, 2020; Accepted November 5, 2020

DOI: $10.3892 / \mathrm{mmr} .2021 .12395$

\begin{abstract}
Gycyrrhizic acid (GA), an inhibitor of high mobility group box 1 (HMGB1), inhibits inflammatory responses and is involved in the occurrence and development of several inflammation-related diseases. However, the role of GA in the atherosclerotic lesions caused by diabetes mellitus (DM) remains unknown. In the present study, Sprague Dawley rats were selected to desi=gn a diabetic atherosclerosis (AS) model. Rats from the DM-AS group were subsequently divided into DM-AS, DM-AS + GA (50 mg/kg) and DM-AS + GA (150 mg/kg) groups. Biochemical analyzers were used to measure levels of blood glucose, fasting insulin, total cholesterol, total triglyceride, low-density lipoprotein and high-density lipoprotein. The number of plaques was recorded after collection of thoracic aortas from the rats. The intimal thickness of arterial tissue was detected by hematoxylin and eosin staining. The expression levels of CD68 and $\alpha$-smooth muscle actin ( $\alpha$-SMA) were detected by immunohistochemistry. The expression of tumor necrosis factor- $\alpha$, interleukin (IL)- 6 and IL- $1 \beta$ in the serum of the rats was detected by ELISA. The expression of fatty acid synthetase, sterol regulatory element binding protein 1C, HMGB1 and receptor for advanced glycation end products (RAGE) was detected by western blotting. Reverse transcription quantitative PCR was used to detect the mRNA expression of
\end{abstract}

Correspondence to: Professor Daimin Zhang, Department of Cardiology, Nanjing First Hospital, Nanjing Medical University, 68 Chang Le Road, Nanjing, Jiangsu 210006, P.R. China

E-mail: zhangdmun@126.com

Abbreviations: $\quad \alpha$-SMA, $\alpha$-smooth muscle actin; AS, atherosclerosis; DM, diabetes mellitus; FINS, fasting insulin; FAS, fatty acid synthetase; GA, gycyrrhizic acid; HDL-C, high-density lipoprotein cholesterol; HMGB1, high mobility group box 1 ; IL-1 $\beta$, interleukin-1 $\beta$; IL-6, interleukin-6; LDL-C, low-density lipoprotein cholesterol; RAGE, receptor for advanced glycation end products; SREBP-1c, sterol regulatory element binding protein $1 C$; TC, total cholesterol; TG, total triglyceride; TNF- $\alpha$, tumor necrosis factor- $\alpha$

Key words: gycyrrhizic acid, diabetes mellitus, atherosclerotic lesions, inflammation
HMGB1 and RAGE. The results demonstrated that GA treatment could decrease the body weight, blood glucose level and biochemical parameters of AS DM rats in a dose-dependent manner. In addition, GA decreased the intimal thickness of carotid artery and the formation of plaque in rats with diabetic AS. Furthermore, GA inhibited macrophage activation and decreased $\alpha$-SMA expression in vascular smooth muscle cells, and decreased the expression of proteins (FAS and SREBP-1c) and inflammatory factors. Taken together, the findings from the present study demonstrated that GA may have a therapeutic effect on DM-associated AS. This study provides a theoretical basis for the treatment of diabetic AS.

\section{Introduction}

Diabetes mellitus (DM) is a group of metabolic diseases characterized by hyperglycemia and divided into type 1 and type 2 diabetes. The two types of DM can be caused by genetic and environmental factors (1). The typical symptoms of DM include polydipsia, polyuria, polyphagia, weight loss, fatigue, weakness and obesity (2). In 2019, 463 million adults aged between 20 and 79 suffered from DM worldwide, with a prevalence rate of about $9.3 \%$ (3). The major long-term and chronic complications of diabetes mellitus (DM) are vascular complications, which are the main causes of increased morbidity and mortality in patients with DM $(4,5)$. Numerous associations between DM and atherosclerosis (AS) have been identified, too much sugar in the blood is associated with insufficient insulin production or a reduced body response to insulin $(6,7)$. Clinical studies have suggested that there is an association between the risk of diabetic vascular complications and poor glycemic control $(8,9)$. AS refers to the buildup of vascular wall lesions caused by chronic inflammation, which result in hypertension, formation of blood clot, strokes, heart attack (10). Hyperglycemia and insulin resistance are important causes of chronic inflammation in patients with DM, therefore accelerating the development of AS (11). In addition, inflammatory factors, such as IL-1 $\beta$ and IL-6, can promote the migration and proliferation of smooth muscle cells to the intima of blood vessels, leading to intima-media thickening and promoting the occurrence and development of AS $(12,13)$.

High mobility group box 1 (HMGB1) is an important mediator of the inflammatory response, which can serve as a pro-inflammatory factor that promotes the occurrence and 
development of inflammation $(14,15)$. HMGB1 overexpression is associated with the pathogenesis of AS (16), obesity (17) and metabolic syndromes (18). Glycyrrhizic acid (GA), an inhibitor of HMGB1, may have a protective effect in AS. Ding et al (19) reported that GA decreases high-fat diet-induced AS in Apoe A- $^{-1}$ mice by significantly decreasing serum HMGB1 and lipid levels, and by increasing the regulatory T-cell/T helper T-cell ratio. In addition, it has been demonstrated that GA can prevent diabetic nephropathy by activating the AMP-activated protein kinase/sirtuin 1/peroxisome proliferator-activated receptor $\gamma$ co-activator 1 signaling pathway in $\mathrm{db} / \mathrm{db}$ mice (20). GA can also decrease the vascular complications induced by diabetic nephropathy (21). However, the effect of GA on DM-associated AS remains unknown. The present study therefore aimed to explore whether GA could decrease HMGB1 expression and reduce the atherosclerotic damage caused by DM.

\section{Materials and methods}

Animal model and treatment. Male Sprague Dawley rats $(\mathrm{n}=40)$ weighing 180-200 g from Changzhou Cavens Laboratory Animal Co. Ltd.) were divided into the control group $(n=10)$ and the diabetic AS group (DM-AS; n=30) and were kept in an environment with a constant temperature of $25^{\circ} \mathrm{C}$ and humidity of $30-70 \%$ with $12 \mathrm{~h}$ light/dark cycle, the mice were fed normally and maintained in specific pathogen-free conditions. All animal procedures and experimental methods were approved by the Committee on the Ethics of Animal Experiments of the Fifth Affiliated Hospital of Zhengzhou University (approval no. 2019A111501) and animal experiments were reported in accordance with the ARRIVE guidelines. After 8 weeks of a high-fat diet, which contained $45.6 \%$ fat, rats in the DM-AS group were injected with low-dose streptozotocin (STZ; 30 mg/kg; Sigma-Aldrich; Merck KGaA) to establish a diabetic AS model (22). The control group was given a normal diet, which contained $\sim 4.0 \%$ fat, and the same dose of citric acid buffer containing STZ was injected intravenously at week 8 . Rats from the DM-AS group were subsequently divided into DM-AS, DM-AS + GA $(50 \mathrm{mg} / \mathrm{kg})$ and DM-AS + GA (150 mg/kg) groups. Once the model was established successfully, the rats were given 50 or $150 \mathrm{mg} / \mathrm{kg} /$ day GA by intragastric administration for 16 weeks. The control and experimental groups were given the same dose of normal saline by gavage. Rats were sacrificed by cervical dislocation following anesthesia with pentobarbital sodium (60 mg/kg intraperitoneal injection). Peripheral blood $(3 \mathrm{ml})$ was collected from the inner canthus of each rat and serum was obtained following centrifugation at $150 \mathrm{x} \mathrm{g}$ for $10 \mathrm{~min}$ at $4^{\circ} \mathrm{C}$ for biochemical assays. Furthermore, aorta specimens were removed carefully and fixed in $4 \%$ paraformaldehyde for hematoxylin and eosin (H\&E) and immunohistochemistry (IHC) staining. The other aorta tissues were stored at $-80^{\circ} \mathrm{C}$ for reverse transcription quantitative PCR (RT-qPCR), western blotting and ELISA.

Biochemical assays. The levels of blood glucose, high-density lipoprotein cholesterol (HDL-C), low-density lipoprotein cholesterol (LDL-C), total cholesterol (TC), total triglycerides (TG) and fasting insulin (FINS) were measured in the serum using an automatic biochemical analyzer (MR-96A;
Mindray Bio-Medical Electronics Co., Ltd.) from the Hospital of Metabolic Disease of Tianjin Medical University (Tianjin, China) according to the manufacturers' instructions.

Histopathological examination. Once mice were euthanized, the chest was cut open after the collection of blood. The aorta was collected quickly and rinsed gently with saline. A 2- to 3-mm slice was cut from the aortic arch and fixed with $4 \%$ paraformaldehyde at $4^{\circ} \mathrm{C}$ for $24 \mathrm{~h}$ and embedded in paraffin. Samples were cut into $4-\mu \mathrm{m}$ sections for H\&E staining for $10 \mathrm{~min}$ at room temperature. Aortic pathology changes were observed using a microscope camera (BA400 Digital; Mike Audi Industrial Group Co., Ltd.). The thickness of the intima and the media of arterial tissue was measured using Image-Pro Plus 6.0 software (Media Cybernetics, Inc.).

IHC staining. Fixed and paraffin-embedded aorta tissue samples were cut into 4- $\mu \mathrm{m}$ sections and were dewaxed. Endogenous peroxidase activity and non-specific binding were blocked by incubation with $3 \%$ hydrogen peroxide and $100 \%$ non-immune serum (closed serum; cat. no. G9023; Sigma-Aldrich; Merck $\mathrm{KGaA}$ ) respectively for $30 \mathrm{~min}$ at room temperature. Sections were incubated with anti-CD68 (EMD Millipore; cat. no. $051050 ; 1: 250$ ) and anti- $\alpha$-SMA (Abcam; cat. no. ab32575; 1:250) antibodies at $4{ }^{\circ} \mathrm{C}$ overnight, and with anti-rabbit secondary antibody (1:5,000; cat. no. ab181658; Abcam) at room temperature for $1 \mathrm{~h}$. Diaminobenzidine hydrochloride (Dako; Agilent Technologies, Inc.) was then added to localize positive staining that was acquired using light microscopy. The sections were counterstained with hematoxylin for $10 \mathrm{~min}$ at room temperature. The data were analyzed via densitometry using ImageJ software (version 146; National Institutes of Health).

Western blotting. Fresh tissues were lysed using RIPA buffer (Sigma-Aldrich; Merck KGaA). Protein concentration was determined using bicinchoninic acid assay protein assay kit. Proteins $(25 \mu \mathrm{g} /$ lane) were separated by $10 \%$ SDS-PAGE and transferred onto PVDF membranes and blocked in 5\% non-fat milk at room temperature for $1 \mathrm{~h}$. Membranes were subsequently incubated with primary antibodies overnight at $4^{\circ} \mathrm{C}$ against fatty acid synthetase (FAS; 1:1,000; cat. no. ab133619; Abcam), sterol regulatory element binding protein 1C (SREBP-1c; 1:1,000; cat. no. PA1-337; Thermo Fisher Scientific, Inc.), HMGB1 (1:1,000; cat. no. ab18256; Abcam), receptor for advanced glycation end products (RAGE; 1:1,000; cat. no. ab216329; Abcam) and GAPDH (1:1,000; cat. no. ab181602; Abcam). Membranes were then incubated with goat anti-rabbit horseradish peroxidase-conjugated secondary antibody (1:5,000; cat. no. ab181658; Abcam) at room temperature for $2 \mathrm{~h}$. Enhanced chemiluminescence reagent (Amersham Pharmacia Biotech Inc.; Cytiva) was used to detect the signal on the membrane. The data were analyzed via densitometry using ImageJ software (version 1.46; National Institutes of Health) and normalized to the expression of the internal control GAPDH.

ELISA. Expression of inflammatory cytokines in serum and tissues were detected by ELISA kits. The levels of IL-6 (cat. no. 506-RL-010), IL-1 $\beta$ (cat. no. 501-RL-010), and TNF- $\alpha$; 

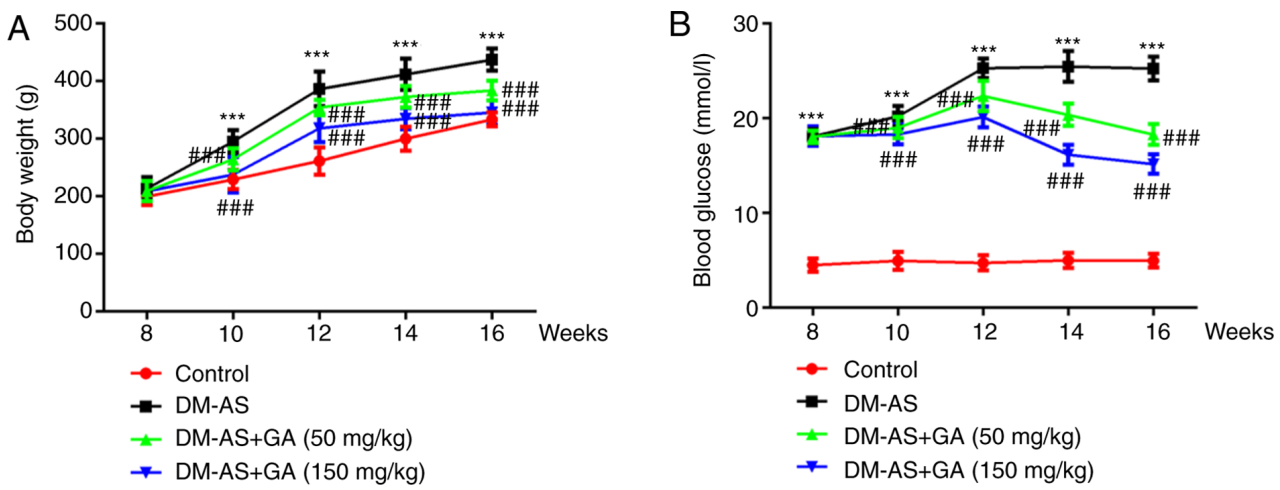

Figure 1. Effects of GA on the body weight and blood glucose level of DM-AS rats. (A) Rat weight and (B) blood glucose level were measured every 2 weeks. ${ }^{* * *} \mathrm{P}<0.001$ vs. control group; ${ }^{\# \#} \mathrm{P}<0.001$ vs. DM-AS group. AS, atherosclerosis; DM, diabetes mellitus; GA, gycyrrhizic acid.

cat. no. 5035-TG-025) were measured using ELISA kits (R\&D Systems, Inc.) according to the manufacturers' instructions.

$R T$ - $q P C R$. Total RNA was isolated from tissues using TRIzol ${ }^{\circledR}$ reagent (Thermo Fisher Scientific, Inc.) according to the suppliers' instructions. cDNA was synthesized using a SuperScript III First-Strand Synthesis SuperMix for qRT-PCR (Thermo Fisher Scientific, Inc.) according to the manufacturers' instructions. SYBR Green PCR Master mix (Applied Biosystems; Thermo Fisher Scientific, Inc.) was used to detect mRNA expression level. The thermocycling conditions were as follows: $95^{\circ} \mathrm{C}$ for $10 \mathrm{~min}, 40$ cycles of $95^{\circ} \mathrm{C}$ for $10 \mathrm{sec}, 55^{\circ} \mathrm{C}$ for $10 \mathrm{sec}$ and $72^{\circ} \mathrm{C}$ for $30 \mathrm{sec}$. GAPDH was used as an internal control. The sequences of the primers were as follows: HMGB1 forward, 5'-GGGATGGCAAAGTTTTTCCCTTTA-3' and reverse 5'-CACTAACCCTGCTGTTCGCT-3'; RAGE forward, 5'-ACAGAAACCGGTGATGAAGG-3' and reverse, 5'-ATT CAGCTCTGCACGTTCCCT-3'; and GAPDH forward, 5'-ATT GTCAGCAATGCATCCTG-3' and reverse 5'-GTAGGCCAT GAGGTCCACdCA-3'. The relative levels of gene expression were quantified by using the comparative CT method (23).

Statistical analysis. SPSS 18.0 (SPSS, Inc.) was used to analyze the results. Data are expressed as the mean \pm standard deviation. Comparisons among multiple groups were analyzed using one-way ANOVA followed by Tukey's post hoc test. $\mathrm{P}<0.05$ was considered to indicate a statistically significant difference.

\section{Results}

Effects of GA on the body weight and serum blood glucose level of DM-AS rats. The weight of the rats was recorded week 8 and every 2 weeks up to week 16 . The results demonstrated that, compared with the control group, the weight of rats in the DM-AS group was significantly increased; however, treatment with GA could decrease rat body weight in a dose-dependent manner (Fig. 1A). During the same period, the serum blood glucose level of rats was assessed, and the results demonstrated that glycemia was significantly increased in the DM-AS group compared with that in the control group. In addition, compared with the DM-AS group, the blood glucose level of rats in the DM-AS + GA group significant decreased from the second week following administration of GA, and this decrease was time- and concentration-dependent (Fig. 1B). Furthermore, the expression levels of HMGB1 and RAGE were significantly increased in the DM-AS group compared with the control group, which was reversed following treatment with GA in a dose-dependent manner (Fig. 2A). These results were similar to those obtained following western blotting (Fig. 2B).

Effects of GA on the biochemical parameters of DM-AS rats. The results demonstrated that, compared with the control group, the levels of FINS, TG, TC and LDL-C (Fig. 3A-C) were significantly increased in the DM-AS group, whereas HDL-C level was significantly decreased (Fig. 3C). However, following DM-AS rat treatment with 50 and $150 \mathrm{mg} / \mathrm{kg} \mathrm{GA}$, the levels of FINS, TG, TC and LDL-C (Fig. 3A-C) were significantly decreased in a dose-dependent manner compared with that in the control group, whereas HDL-C level was significantly increased (Fig. 3C).

Effects of GA on aortic plaques in DM-AS rats. Aortic plaques were observed in the rats and the results demonstrated that plaque number was increased in rats from the DM-AS group compared with that in rats in the control group. Compared with that in the DM-AS group, the percentage of aortic plaques in the DM-AS + GA (50 mg/kg) and DM-AS + GA $(150 \mathrm{mg} / \mathrm{kg}$ ) groups was decreased in a dose-dependent manner (Fig. 4A and C). Furthermore, the intima thickness of arterial tissues was observed by H\&E staining, and the results demonstrated that the intima thickness of arterial tissues in the DM-AS group was significantly increased compared with that in the control group. However, following treatment with GA (50 or $150 \mathrm{mg} / \mathrm{kg}$ ), intima thickness was decreased in a concentration-dependent manner (Fig. 4B and D). These findings suggested that GA may reduce the intimal thickness of the aorta and the formation of plaques in DM-AS rats in a dose-dependent manner.

Effects of GA on macrophage activation, $\alpha-S M A$ expression and lipid factors (FAS and SREBP-1c) expression in $D M-A S$ rats. IHC was performed to detect the expression of CD68 and $\alpha$-SMA in aorta tissues from rats. As presented in Fig. 5, the expression of CD68 and $\alpha$-SMA was significantly increased in the DM-AS group compared with that in the control group. In addition, following treatment with GA, the expression of CD68 and $\alpha$-SMA was significantly 

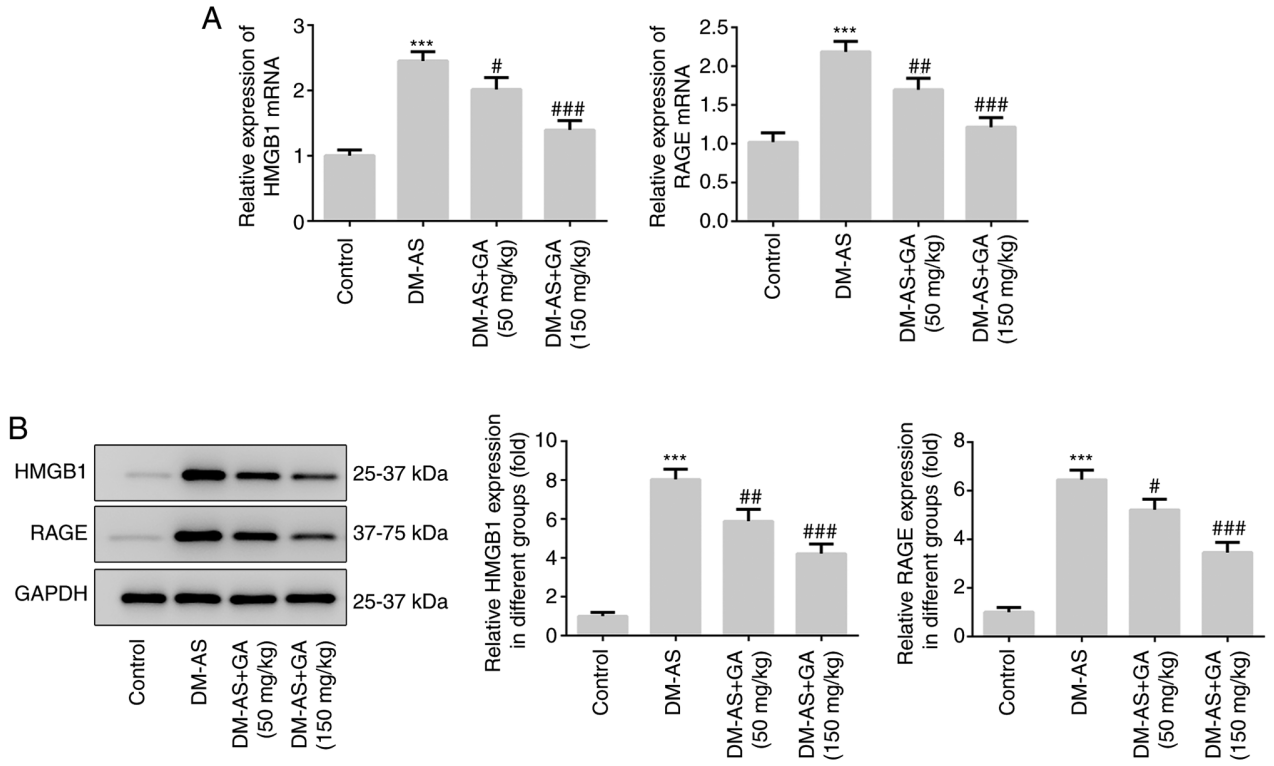

Figure 2. Expression of HMGB1 and RAGE. (A) Reverse transcription quantitative PCR was used to detect the mRNA expression levels of HMGB1 and RAGE. (B) Western blotting was used to detect the protein expression levels of HMGB1 and RAGE. ${ }^{* * *} \mathrm{P}<0.001$ vs. control group; ${ }^{*} \mathrm{P}<0.05$, ${ }^{\# \#} \mathrm{P}<0.01$ and

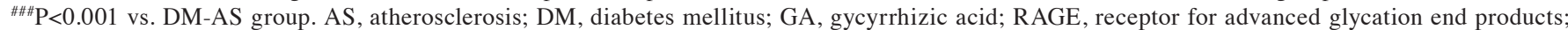
HMGB1, high mobility group box 1 .
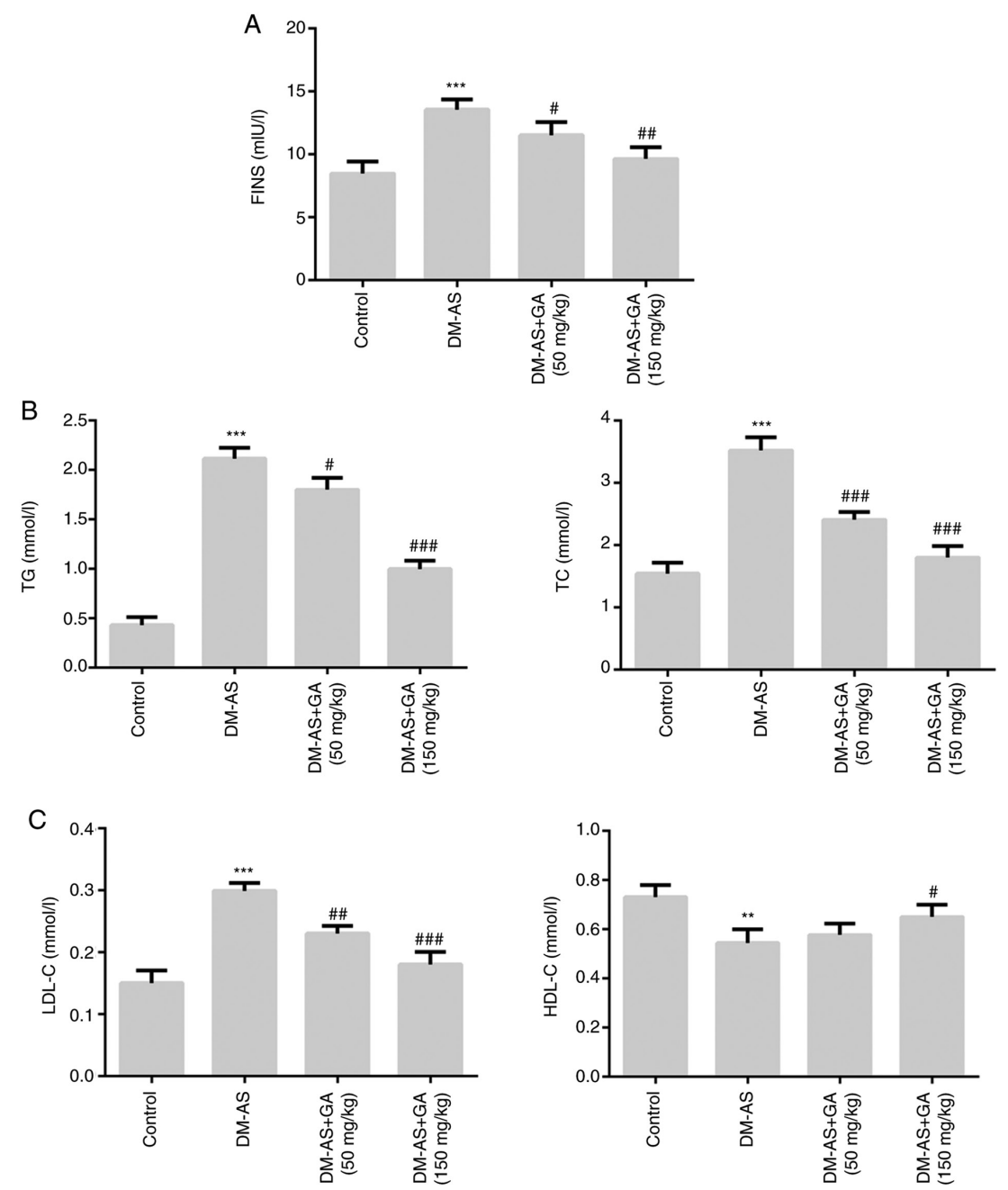

Figure 3. Effects of GA on biochemical parameters in DM-AS rats. Levels of (A) FINS, (B) TG and TC, (C) LDL-C and HDL-C were measured using a biochemical analyzer. ${ }^{* *} \mathrm{P}<0.01,{ }^{* * *} \mathrm{P}<0.001$ vs. control group; ${ }^{\#} \mathrm{P}<0.05,{ }^{\# \#} \mathrm{P}<0.01$ and ${ }^{\# \# "} \mathrm{P}<0.001$ vs. DM-AS group. AS, atherosclerosis. AS, atherosclerosis; DM, diabetes mellitus; GA, gycyrrhizic acid; FINS, fasting insulin; HDL-C, high density lipoprotein cholesterol; LDL-C, low density lipoprotein cholesterol; TC, total cholesterol; TG, total triglyceride. 
A

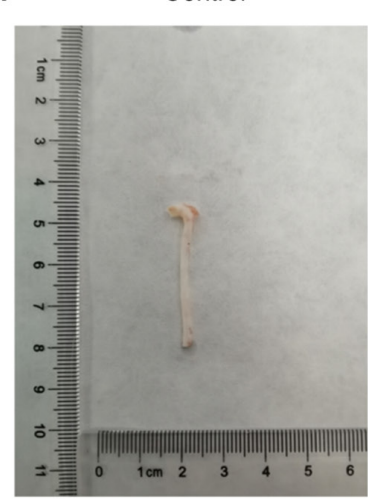

B
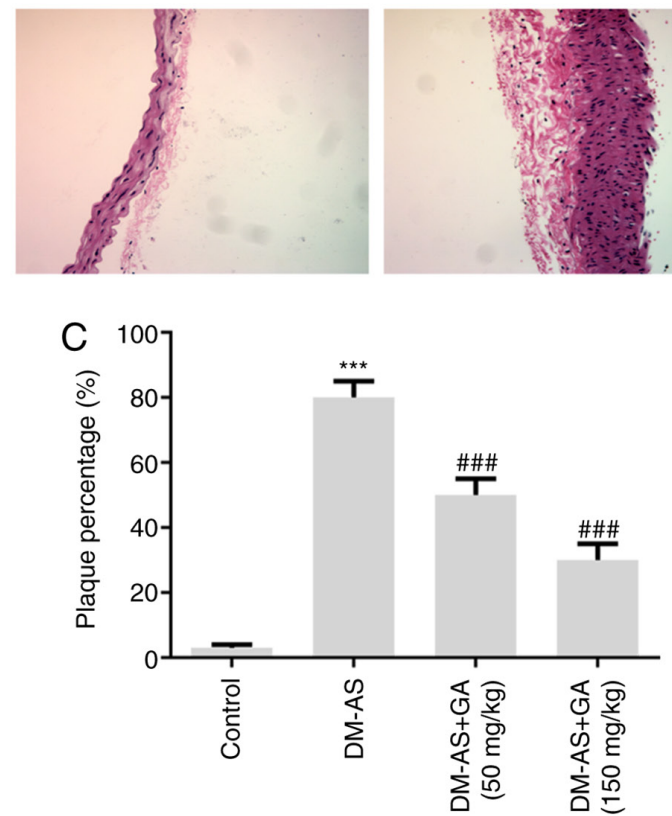

DM-AS
DM-AS+GA $(50 \mathrm{mg} / \mathrm{kg})$
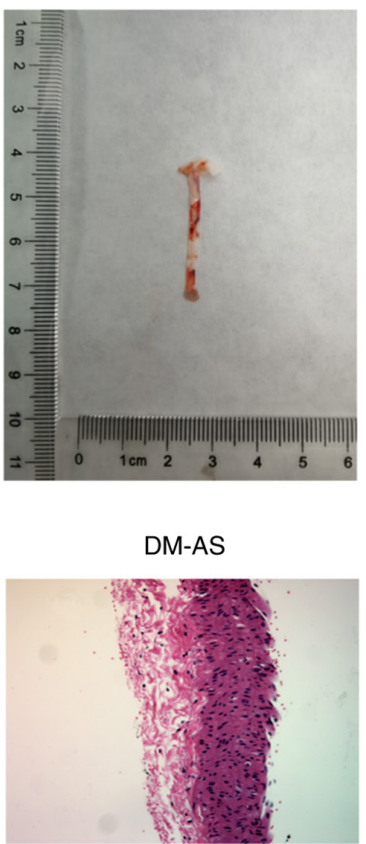

DM-AS+GA (50 mg/kg)

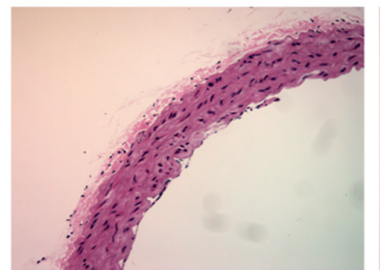

DM-AS+GA (150 mg/kg)

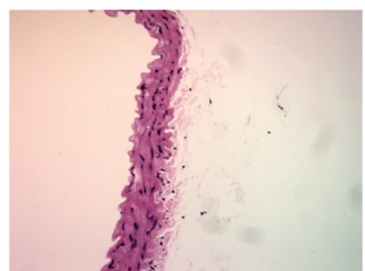

D

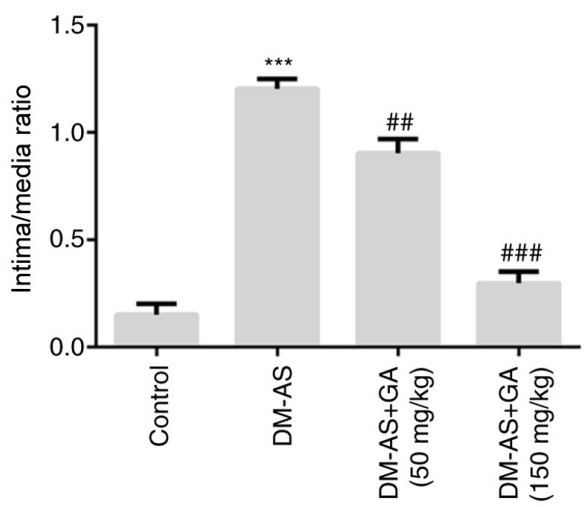

Figure 4. Effects of GA on plaque formation in DM-AS rats. (A) Images of aortas from rats. (B) Hematoxylin and eosin staining was used to detect the thickness of intima and media of arterial tissue (magnification, x200). (C) Percentage of plaque formation. (D) Ratio of intima/media thickness. ${ }^{* * *} \mathrm{P}<0.001$ vs. control group; ${ }^{\# \#} \mathrm{P}<0.01$ and ${ }^{\# \# \#} \mathrm{P}<0.001$ vs. DM-AS group. AS, atherosclerosis; DM, diabetes mellitus; GA, gycyrrhizic acid.

decreased (Fig. 5A and B). Subsequently, the expression of lipid metabolism-related proteins FAS and SREBP-1c was detected by western blotting. The expression of FAS and SREBP-1c increased in the DM-AS group compared with that in the control group. In addition, following treatment with GA, the expression of FAS and SREBP-1c was also significantly decreased (Fig. 5C).

Effects of GA on the concentration of inflammatory factors in serum and atherosclerotic tissue of DM-AS rats. The concentrations of inflammatory cytokines were measured in the serum (Fig. 6A) and atherosclerotic tissue (Fig. 6B) of rats. Compared with that of the control group, the expression of TNF- $\alpha$, IL- 6 and IL- $1 \beta$ in the DM-AS group was significantly increased. In addition, the expression of TNF- $\alpha$, IL-6, and IL-1 $\beta$ in the DM-AS + GA $(50 \mathrm{mg} / \mathrm{kg})$ and DM-AS + GA $(150 \mathrm{mg} / \mathrm{kg})$ groups was significantly decreased in a dose-dependent manner compared with that in the DM-AS group. These findings suggested that GA may reduce inflammation in DM-AS rats.

\section{Discussion}

Cardiovascular disease is a common complication that can be fatal in people with DM. Previous studies have demonstrated that cardiovascular diseases account for $>50 \%$ of mortality cases in patients with DM, with ischemia, angina, myocardial infarction, stroke and sudden cardiac death caused by AS accounting for a large proportion of these cases $(9,24)$. In the diabetic population, the incidence of AS is higher, the disease is more serious and the mortality rate is increased compared with the non-diabetic population (25). Coronary heart disease, myocardial infarction and acute cerebrovascular disease are the main causes of mortality in patients with DM (26). Therefore, whether cardiovascular diseases could be prevented by decreasing blood glucose level has become a focus of interest.

In the present study, rats were fed with a high-fat diet for 8 weeks and injected with STZ to establish a DM-AS model (22). The blood glucose level and DM-related indexes in DM-AS rats were significantly increased compared with those of the control group. Furthermore, the number of arterial 

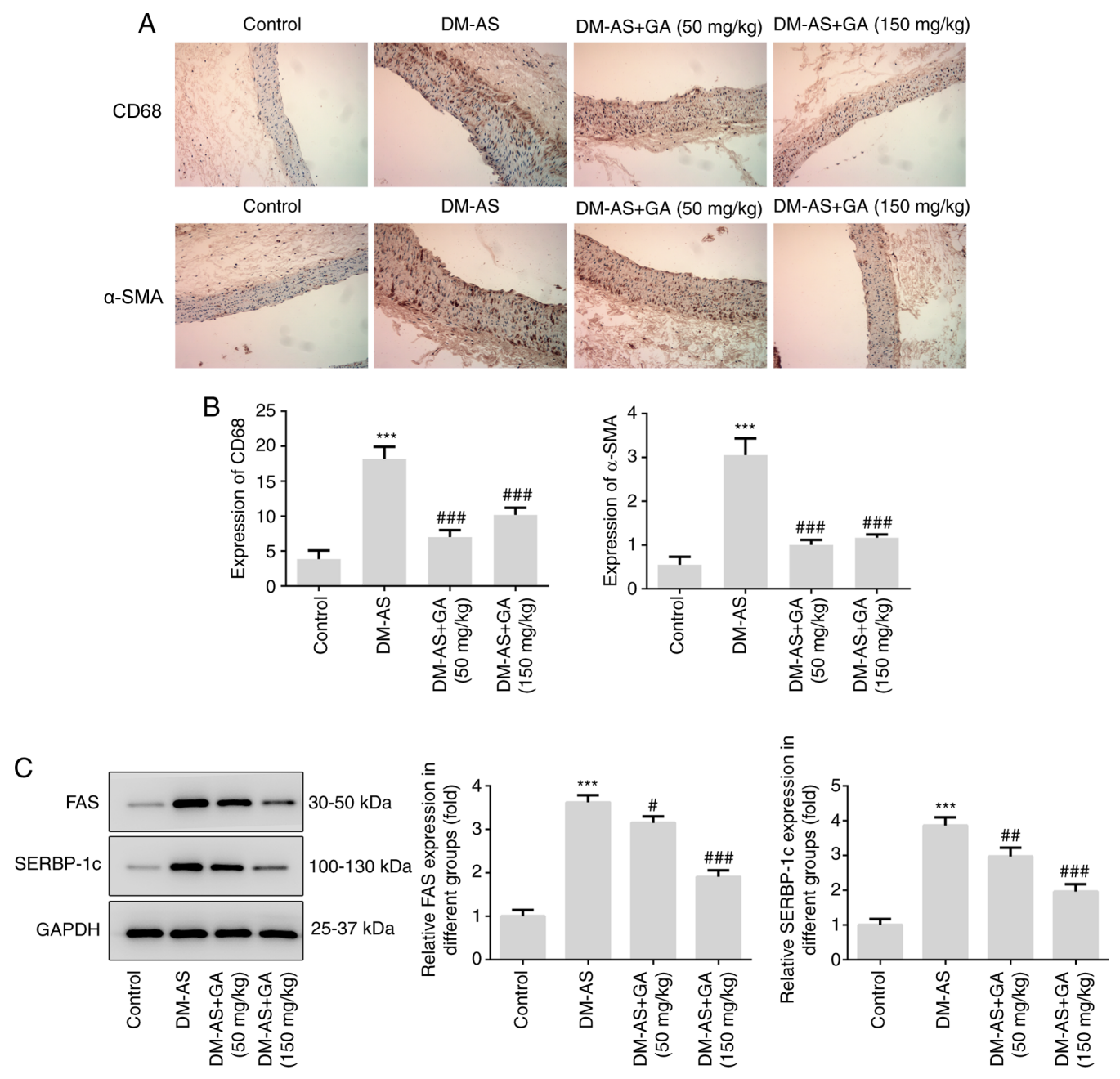

Figure 5. Effects of GA on macrophage activation, $\alpha$-SMA expression, FAS and SREBP-1c expression in DM-AS rats. (A) Immunohistochemistry was used to detect CD68 and $\alpha$-SMA expression in aortic tissues (magnification, x200). (B) Quantitative analysis of CD68 and $\alpha$-SMA expression from (A). (C) Western blotting detected the expression of FAS and SREBP-1c. ${ }^{* * *} \mathrm{P}<0.001$ vs. control group; ${ }^{\#} \mathrm{P}<0.05,{ }^{\# \#} \mathrm{P}<0.01$ and ${ }^{\# \# \#} \mathrm{P}<0.001$ vs. DM-AS group. AS, atherosclerosis; DM, diabetes mellitus; GA, gycyrrhizic acid; $\alpha$-SMA, $\alpha$-smooth muscle actin; SREBP-1c, sterol regulatory element binding protein $1 C$.
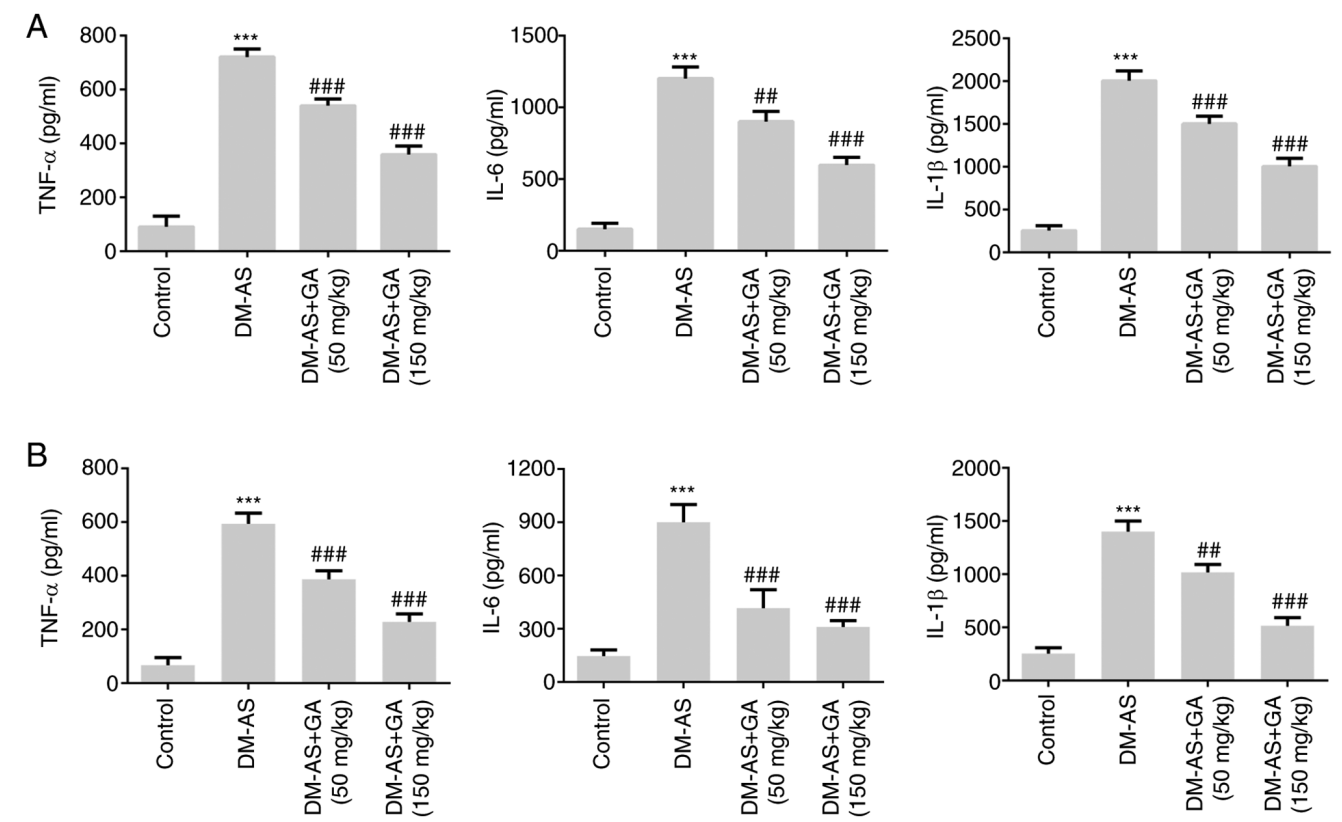

Figure 6. Effects of GA on the levels of inflammatory factors in the serum and aortas of DM-AS rats. ELISA was used to detect the concentration of TNF- $\alpha$, IL- 6 and IL-1 $\beta$ in the (A) serum and (B) aortas of rats. ${ }^{* * *} \mathrm{P}<0.001$ vs. control group; ${ }^{* \#} \mathrm{P}<0.01$ and ${ }^{\# \# \#} \mathrm{P}<0.001$ vs. DM-AS group. AS, atherosclerosis; DM, diabetes mellitus; GA, gycyrrhizic acid; TNF- $\alpha$, tumor necrosis factor- $\alpha$; IL-1 $\beta$, interleukin-1 $\beta$; IL-6, interleukin-6. 
plaques, intima thickness, macrophage activation and $\alpha$-SMA expression in DM-AS rats were increased compared with those in the control rats, indicating the successful establishment of the DM-AS model. It was found that the normal blood glucose level of rats is $\sim 4 \mathrm{mmol} / \mathrm{l}$ (27), similar to the results observed in the present study.

The present study mainly evaluated whether GA could decrease blood glucose level in patients with DM and improve renal injury caused by DM (28). In the present study, GA treatment decreased blood glucose, insulin, TC, TG and LDL-C levels, and stimulated the formation of HDL-C in DM-AS rats. The typical pathological features of AS are increased arterial plaque number, a thickened arterial intima, activated macrophages, increased lipid metabolism and aggravated inflammatory reactions (29). In the present study, GA treatment decreased the number of atherosclerotic artery plaques in DM-AS rats in a dose-dependent manner, reduced the intima thickness, inhibited the activation of macrophages, and alleviated the inflammatory response and lipid metabolism. These findings suggested that GA may have some therapeutic effects on DM-AS. GA is a small inhibitor of HMGB1 that has a protective effect on blood vessels in AS $(19,30)$ and can decrease renal complications caused by DM (21). In the present study, GA was found to inhibit the expression of HMGB1 in DM-AS rats. Furthermore, RAGE is one of the important receptors of HMGB1. When combined, the two receptors activate the intracellular signal transduction pathway, leading to pathological damage and affecting the pathogenesis of inflammatory diseases, such as arthritis and AS (31). In the present study, GA was demonstrated to also inhibit the expression of RAGE in DM-AS rats.

SREBP-1c coordinates the synthesis of FA and cholesterol, and FAS is its downstream transcription factor (32). A previous study has reported that GA can improve lipid metabolism in AS and inhibit vascular inflammation in Apoe ${ }^{-/}$mice (19). The results from the present study demonstrated that GA could inhibit the expression of the lipid-related proteins FAS and SREBP-1c in DM-AS rats. It has been reported that GA has some inhibitory effects on neointimal hyperplasia in rat models of common carotid artery injury (33). Similarly, the present study demonstrated that GA could decrease the intima thickness of arterial tissues in DM-AS rats. Furthermore, it is well known that macrophages serve a crucial role in the formation of atherosclerotic plaques, and CD68 is considered as a marker of macrophage activation (34). In the present study, a significant decrease in the expression of CD68 in DM-AS rats treated with GA was observed.

In summary, the findings from this study suggested that GA may improve atherosclerotic injury caused by DM and provide a theoretical basis for the treatment of DM-AS.

\section{Acknowledgements}

Not applicable.

\section{Funding}

This study was funded by the Provincial Key Research and Development Program (grant no. BE2018611) and the Science and Technology Foundation of Henan Province (grant no. 112102310189).

\section{Availability of data and materials}

The datasets used and/or analyzed during the current study are available from the corresponding author on reasonable request.

\section{Authors' contributions}

DZ made substantial contributions to the conception and design of the study, and the acquisition of data. YZ and WL made substantial contributions to analysis and interpretation of data. YZ and DZ confirm the authenticity of all the raw data. All authors read and approved the final manuscript.

\section{Ethics approval and consent to participate}

The study protocol was approved by the Ethics Committee of The Fifth Affiliated Hospital of Zhengzhou University. All animal experiments complied with the ethical requirements of the animal council.

\section{Patient consent for publication}

Not applicable.

\section{Competing interests}

The authors declare that they have no competing interests.

\section{References}

1. Guthrie RA and Guthrie DW: Pathophysiology of diabetes mellitus. Crit Care Nurs Q 27: 113-125, 2004

2. Unnikrishnan R, Anjana RM and Mohan V: Diabetes mellitus and its complications in India. Nat Rev Endocrinol 12: 357-370, 2016.

3. Saeedi P,Petersohn I, Salpea P, Malanda B, Karuranga S, Unwin N, Colagiuri S, Guariguata L, Motala AA, Ogurtsova K, et al: Global and regional diabetes prevalence estimates for 2019 and projections for 2030 and 2045: Results from the International Diabetes Federation Diabetes Atlas, 9(th) edition. Diabetes Res Clin Pract 157: 107843, 2019.

4. Strain WD and Paldánius PM: Diabetes, cardiovascular disease and the microcirculation. Cardiovasc Diabetol 17: 57, 2018.

5. Kannenkeril D, Bosch A, Harazny J, Karg M, Jung S, Ott C and Schmieder RE: Early vascular parameters in the micro- and macrocirculation in type 2 diabetes. Cardiovasc Diabetol 17: 128,2018

6. Di Pino A and DeFronzo RA: Insulin resistance and atherosclerosis: Implications for insulin-sensitizing agents. Endocr Rev 40: 1447-1467, 2019.

7. Stabley JN and Towler DA: Arterial calcification in diabetes mellitus: Preclinical models and translational implications. Arterioscler Thromb Vasc Biol 37: 205-217, 2017.

8. Poznyak A, Grechko AV, Poggio P, Myasoedova VA, Alfieri V and Orekhov AN: The diabetes mellitus-atherosclerosis connection: The role of lipid and glucose metabolism and chronic inflammation. Int J Mol Sci 21: 1835, 2020.

9. Ioachimescu AG: Diabetes and atherosclerotic cardiovascular disease. Endocrinol Metab Clin North Am 47: xiii-xiv, 2018.

10. Zhu Y, Xian X, Wang Z, Bi Y, Chen Q, Han X, Tang D and Chen R: Research progress on the relationship between atherosclerosis and inflammation. Biomolecules 8: 80, 2018.

11. Laakso $\mathrm{M}$ and Kuusisto J: Insulin resistance and hyperglycaemia in cardiovascular disease development. Nat Rev Endocrinol 10: 293-302, 2014.

12. Yahagi K, Kolodgie FD, Lutter C, Mori H, Romero ME, Finn AV and Virmani R: Pathology of human coronary and carotid artery atherosclerosis and vascular calcification in diabetes mellitus. Arterioscler Thromb Vasc Biol 37: 191-204, 2017. 
13. Dryden M, Baguneid M, Eckmann C, Corman S, Stephens J, Solem C, Li J, Charbonneau C, Baillon-Plot N and Haider S: Pathophysiology and burden of infection in patients with diabetes mellitus and peripheral vascular disease: Focus on skin and softtissue infections. Clin Microbiol Infect 21 (Suppl 2): S27-S32, 2015

14. Malahfji M and Mahmarian JJ: Imaging to stratify coronary artery disease risk in asymptomatic patients with diabetes. Methodist Debakey Cardiovasc J 14: 266-272, 2018.

15. Andersson U and Tracey KJ: HMGB1 is a therapeutic target for sterile inflammation and infection. Annu Rev Immunol 29 : 139-162, 2011.

16. Deng M, Scott MJ, Fan J and Billiar TR: Location is the key to function: HMGB1 in sepsis and trauma-induced inflammation. J Leukoc Biol 106: 161-169, 2019.

17. Wang R, Wu W, Li W, Huang S, Li Z, Liu R, Shan Z, Zhang C, Li W and Wang S: Activation of NLRP3 inflammasome promotes foam cell formation in vascular smooth muscle cells and atherogenesis Via HMGB1. J Am Heart Assoc 7: e008596, 2018.

18. Zhang J, Zhang L, Zhang S, Yu Q, Xiong F, Huang K, Wang CY and Yang P: HMGB1, an innate alarmin, plays a critical role in chronic inflammation of adipose tissue in obesity. Mol Cell Endocrinol 454: 103-111, 2017.

19. Ding JW, Luo CY, Wang XA, Zhou T, Zheng XX, Zhang ZQ, $\mathrm{Yu}$ B, Zhang J and Tong XH: Glycyrrhizin, a high-mobility group box 1 inhibitor, improves lipid metabolism and suppresses vascular inflammation in apolipoprotein e knockout mice. J Vasc Res 55: 365-377, 2018

20. Hou S, Zhang T, Li Y, Guo F and Jin X: Glycyrrhizic acid prevents diabetic nephropathy by activating AMPK/SIRT1/PGC-10 Signaling in db/db Mice. J Diabetes Res 2017: 2865912, 2017.

21. Zhang H, Zhang R, Chen J, Shi M, Li W and Zhang X: High Mobility Group Box1 inhibitor glycyrrhizic acid attenuates kidney injury in streptozotocin-induced diabetic rats. Kidney Blood Press Res 42: 894-904, 2017.

22. Li J, Liu X, Fang Q, Ding M and Li C: Liraglutide attenuates atherosclerosis via inhibiting ER-induced macrophage derived microvesicles production in T2DM rats. Diabetol Metab Syndr 9: 94, 2017.

23. Livak KJ and Schmittgen TD: Analysis of relative gene expression data using real-time quantitative PCR and the 2(-Delta Delta C(T)) method. Methods 25: 402-408, 2001.

24. Shah AD, Langenberg C, Rapsomaniki E, Denaxas S, Pujades-Rodriguez M, Gale CP, Deanfield J, Smeeth L, Timmis A and Hemingway H: Type 2 diabetes and incidence of cardiovascular diseases: A cohort study in 1.9 million people. Lancet Diabetes Endocrinol 3: 105-113, 2015.
25. Haas AV and McDonnell ME: Pathogenesis of cardiovascular disease in diabetes. Endocrinol Metab Clin North Am 47: 51-63, 2018.

26. Buszman PP, Bochenek A, Konkolewska M, Trela B, Kiesz RS, Wilczyński M, Cisowski M, Krejca M, Banasiewicz-Szkróbka I, Krol M, et al: Early and long-term outcomes after surgical and percutaneous myocardial revascularization in patients with non-ST-elevation acute coronary syndromes and unprotected left main disease. J Invasive Cardiol 21: 564-569, 2009.

27. Zhou J, Zhe R, Guo X, Chen Y, Zou Y, Zhou L and Wang Z: The Role of PPARס Agosnist GW501516 in rats with gestational diabetes mellitus. Diabetes Metab Syndr Obes 13: 2307-2316, 2020.

28. Rani R, Dahiya S, Dhingra D, Dilbaghi N, Kaushik A, Kim KH and Kumar S: Antidiabetic activity enhancement in streptozotocin + nicotinamide-induced diabetic rats through combinational polymeric nanoformulation. Int J Nanomedicine 14: 4383-4395, 2019.

29. Moroni F, Ammirati E, Norata GD, Magnoni M and Camici PG: The role of monocytes and macrophages in human atherosclerosis, plaque neoangiogenesis, and atherothrombosis. Mediators Inflamm 2019: 7434376, 2019.

30. Palone F, Pasquali E, Giardullo P, Stronati L, Vitali R and Mancuso M: Low dose of dipotassium glycyrrhizate counteracts atherosclerosis progression in apoe-/-female mice. J Vasc Res 56: 267-270, 2019.

31. Wang Y, Le Y, Zhao W, Lin Y, Wu Y, Yu C, Xiong J, Zou F, Dong H, Cai S and Zhao H: Short thymic stromal lymphopoietin attenuates toluene diisocyanate-induced airway inflammation and inhibits high mobility group box 1-receptor for advanced glycation end products and long thymic stromal lymphopoietin expression. Toxicol Sci 157: 276-290, 2017.

32. Brown MS and Goldstein JL: The SREBP pathway: regulation of cholesterol metabolism by proteolysis of a membrane-bound transcription factor. Cell 89: 331-340, 1997.

33. Chen J, Zhang J, Xu L, Xu C, Chen S, Yang J and Jiang H: Inhibition of neointimal hyperplasia in the rat carotid artery injury model by a HMGB1 inhibitor. Atherosclerosis 224: 332-339, 2012.

34. Cybulsky MI, Cheong C and Robbins CS: Macrophages and dendritic cells: Partners in atherogenesis. Circ Res 118: 637-652, 2016.

This work is licensed under a Creative Commons Attribution-NonCommercial-NoDerivatives 4.0 International (CC BY-NC-ND 4.0) License. 